\title{
Successful surgical repair and recovery in a 2-week-old infant after birth-related cervical fracture dislocation
}

\author{
Sara Saleh, BS, ${ }^{1}$ Kyle I. Swanson, MD, ${ }^{1,2}$ and Taryn Bragg, MD1,3 \\ 1Department of Neurological Surgery, University of Wisconsin School of Medicine and Public Health, Madison, Wisconsin; \\ 2Department of Neurosurgery, The Barrow Neurological Institute; and 'Division of Neurosurgery, Barrow Neurological Institute at \\ Phoenix Children's Hospital, Phoenix, Arizona
}

\begin{abstract}
Cervical spine injuries are the most common spine injuries in the pediatric population. The authors present the youngest known patient who underwent cervical spine fusion to repair birth trauma-induced cervical fracture dislocation, resulting in spondyloptosis and spinal cord injury. A 2-week-old boy was found to have spondyloptosis and spinal cord injury after concerns arose from reduced movement of the extremities. The patient's birth was complicated by undiagnosed abdominal dystocia, which led to cervical distraction injury. At 15 days of age, the boy underwent successful C-5 corpectomy, with anterior C4-6 and posterior C2-7 arthrodesis, using an autologous rib graft for a C-5 fracture dislocation. MRI performed 2 weeks postoperatively revealed significant improvement in the alignment of the spinal canal. The patient was discharged from the hospital in a custom Minerva brace and underwent close follow-up in addition to occupational therapy and physical therapy. At the latest follow-up 4.5 years later, the patient was able to walk and ride a tricycle by himself. The authors describe the patient's surgery and the challenges faced in achieving successful repair and cervical spine stabilization in such a young patient. The authors suggest that significant neurological recovery after spinal cord injury in infants is possible with appropriate, timely, and interdisciplinary management.
\end{abstract}

https://thejns.org/doi/abs/10.3171/2017.7.PEDS17105

KEY WORDS cervical trauma; spine; neonate; pediatric neurosurgery

A LTHOUGH cervical spine injury is the most common spinal injury in the pediatric population, reports of such injuries during delivery are likely underreported, and the true incidence is unknown., ${ }^{9,22}$ Such cases are associated with high incidences of morbidity and mortality, including paralysis and, in extreme cases, quadriplegia. ${ }^{5,7,14,16,18,20}$ Past reports of successful cervical repair have been made but are few, and complications, including death, are not uncommon. ${ }^{10,11,13,24}$ We report on cervical spine fusion repair in a 2-week-old infant (to our knowledge, the youngest known patient to undergo this procedure) with successful restoration of function. We describe the surgical approaches taken, in addition to the challenges faced in such a young patient with multiple comorbidities. Finally, we discuss the valuable postoperative management in his successful recovery, in addition to findings during his latest follow-up examination at 4.5 years of age.

\section{Case Report}

History and Examination

This baby boy was born at 38 weeks' gestation after an uncomplicated prenatal course. The last gestational ultrasonography study was performed at 20 weeks, the results of which were found to be normal. The patient's birth was complicated by previously undiagnosed abdominal dystocia, which required significant force applied to the patient's head and neck for extraction. The abdominal dystocia was 
due to posterior urethral valves resulting in severe bilateral hydronephrosis and left-sided kidney rupture causing significant abdominal ascites. The posterior urethral valves were corrected surgically shortly after birth. The patient began receiving parenteral nutrition at the beginning of life because surgeries and procedures for the repair of his posterior urethral valves and ascites, respectively, were required. The patient showed poor oral intake upon starting feedings afterward.

The patient's cervical spine injury was identified when he was 14 days old after sagittal MRI revealed C4-5 spondyloptosis, with the body of C-5 being completely anterior to the vertebral body of $\mathrm{C}-4$ and imaging findings concerning for spinal cord transection at that level. MRI was performed after nurses noted decreased movement in the lower extremities. On recognition of the injury, cervical immobilization was instituted using sandbags placed on either side of the patient's head to prevent motion, as the available cervical collars were all too large. The patient was not placed flat on his back, since the relatively large size of an infant's head can result in significant cervical flexion if the infant is placed supine on a flat surface. When supine, padding underneath the body can be used to maintain neutral alignment of the cervical spine. ${ }^{2}$ The patient underwent intubation prior to arrival due to meconium aspiration at delivery and atelectasis of the left lung after the correction of his posterior urethral valves. He remained on a ventilator during his entire hospitalization. He was receiving nutrition via a nasogastric tube on transfer due to continued poor oral intake.

On examination, no obvious deformity was present in the neck or back. The patient's cranial nerves were found to be intact. He moved all 4 extremities spontaneously but weakly. He had notable movement of the deltoid and trapezius muscles bilaterally. The left arm revealed very weak wrist extension and no biceps or left-hand movements. The right arm showed very weak biceps movement, with 1-2/5 strength and 2/5 triceps movement, as well as weak $2 / 5$ wrist extension. No right-hand or finger movement was seen. The patient exhibited increased tone with elbow flexion on the right side, with normal to low tone on the left side.

The lower extremities appeared to spontaneously have weak, 2-3/5 hip flexion, knee flexion, and dorsiflexion, which most likely represented an abnormal triple spinal reflex. Plantar stimulation resulted in a positive Babinski sign bilaterally. The patient exhibited sustained bilateral clonus, which supported the cervical spinal lesion as a likely cause of the movement deficits.

Sensory examination did not reveal any definite reaction to tactile painful stimulation to the hands, forearms, or legs except along the plantar surface of the foot, which caused a triple flexion response. Over the shoulders, the patient exhibited some withdrawal-like movement, suggesting sensation at the level of the shoulders.

\section{Imaging}

Lateral radiography of the cervical spine revealed complete anterior dislocation of the C-5 vertebral body relative to C-4, without evidence of facet locking (Fig. 1 left).

Sagittal T2-weighted MRI revealed bilateral C-5 pedi-
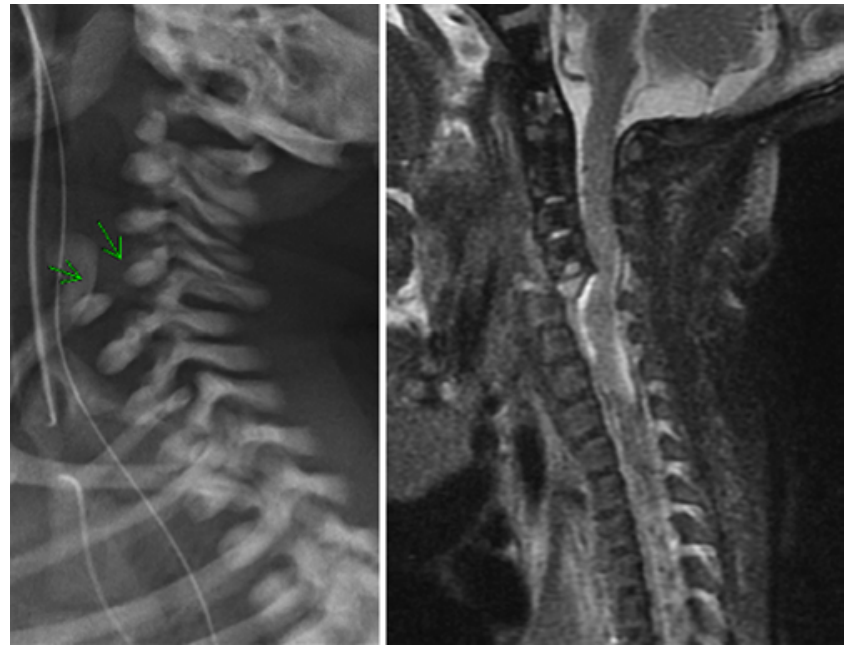

FIG. 1. Left: Preoperative lateral cervical radiograph demonstrating C4-5 cervical spondyloptosis with the C-5 vertebral body anterior to the C-4 vertebral body as a result of distracted bilateral C- 5 pedicle fractures (arrows). Right: Preoperative sagittal T2-weighted MR image confirming complete anterior displacement of the $\mathrm{C}-5$ vertebral body relative to the $\mathrm{C}-4$ vertebral body with severe cervical spinal canal compromise resulting in $\mathbf{C} 4-5$ spinal cord compression and injury. Figure is available in color online only.

cle fractures resulting in spondyloptosis with the C-5 vertebral body anterior to that of $\mathrm{C}-4$, resulting in anterior displacement and kinking of the cervical spinal cord with suspected spinal cord compression at $\mathrm{C} 4-5$ (Fig. 1 right). In addition, a cerebellar subdural hematoma and a small amount of intraventricular hemorrhage were noted. MR angiography of the neck revealed no evidence for flow in the cervical portion of the left vertebral artery. The right vertebral artery was normal in appearance.

\section{Surgical Management}

The patient was taken to the operating room on Day 15 of life and underwent an attempted closed reduction of the dislocation under intraoperative fluoroscopic guidance, along with fitting for a custom Minerva brace (Fig. 2). The closed reduction was unsuccessful, so the next day the patient was returned to the operating room for operative reduction and fusion. This time, he underwent an open reduction via an anterior cervical C-5 corpectomy and fusion using an autologous rib interbody graft and a miniabsorbable plate (KLS Martin) between C-4 and C-6, and a C2-7 posterior cervical fusion using bilateral split autologous rib grafts sutured to the laminae at each level.

We began with an anterior approach with the patient supine. The incision was marked from the $\mathrm{C} 4-5$ disc space medial to the edge of the sternocleidomastoid muscle. The anterior vertebral bodies were identified after developing a plane of blunt dissection, and the C4-5 step-off was clearly identified. We performed the C4-5 discectomy at this time, and unsuccessfully attempted open reduction. Therefore, we elected to proceed with posterior fusion, and the patient was placed prone in his custom Minerva brace after closure of the anterior incision. After performing an autologous rib harvest, we exposed C-2 to C-7 and secured the decorticated harvested ribs to the laminae bilaterally with 

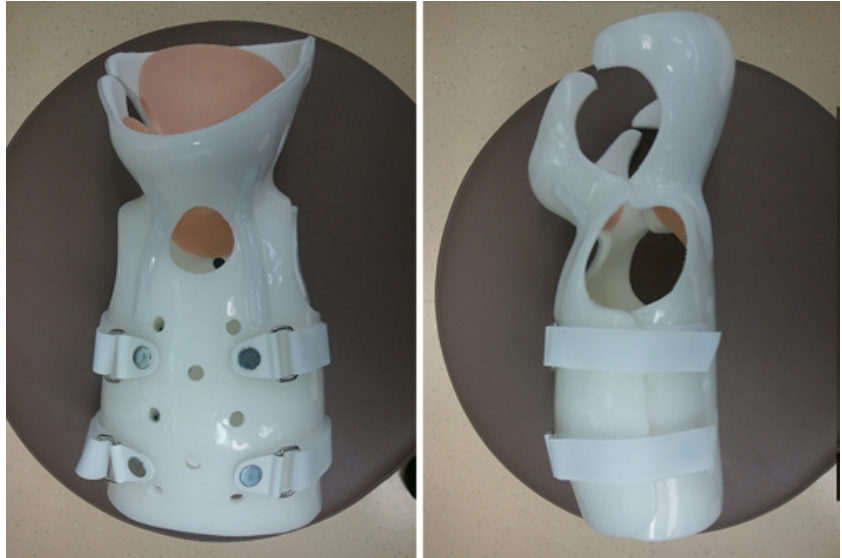

FIG. 2. Photographs of the custom Minerva orthosis made for this patient. Note the high collar surrounding the occiput and mandible to fully immobilize the cervical spine, along with the anterior openings for a tracheostomy tube and ventilation. Figure is available in color online only.

silk suture. This achieved some lordosis but did not significantly reduce the previous $\mathrm{C}-5$ pedicle fractures. However, the laminae were well approximated given the fractures, which had completely disconnected the C-5 vertebral body from the posterior elements. The baby was then placed supine, after which the previous incision was exposed, and the C-5 vertebral body and C5-6 disc space were identified. At this time, we determined that a C-5 corpectomy would be required as the $\mathrm{C}-5$ vertebral body was anterior to C-4 and C-6. We performed the corpectomy using a 1-mm diamond bur and rongeurs from the otolaryngology pan because of the small size of the neonatal cervical spine. A small piece of rib previously harvested for the posterior fusion had been saved to be used as a graft and was fitted perfectly between $\mathrm{C}-4$ and C- 6 . We then placed a miniabsorbable plate from C-4 to C-6.

Somatosensory evoked potentials of the bilateral posterior tibial and median nerves were monitored throughout the operation. Only the right median nerve responses were consistent and therefore used. Toward the end of the cervical fusion, there was a period during which the monitoring changed, but was immediately corrected by transfusing blood.

\section{Postoperative Course}

Postoperatively, nursing staff reported that the patient appeared to have some purposeful movement of the upper extremities, in addition to sensation to light touch. On our examination, he was found to have reduced tone in the upper extremities. He had increased tone in the lower extremities, which remained at discharge. No spontaneous movement was noted immediately postoperatively on our examination, however. No changes were noted on examination of the cranial nerves. Of note, the patient developed seizures an hour after the operation, possibly due to intraoperative blood transfusion. They were self-limited and only recurred once after discharge.

Postoperative MRI confirmed significant improvement in the alignment of the spinal canal with decompression of the spinal cord (Fig. 3 left), and MR angiography demonstrated a newly patent left vertebral artery without evi-
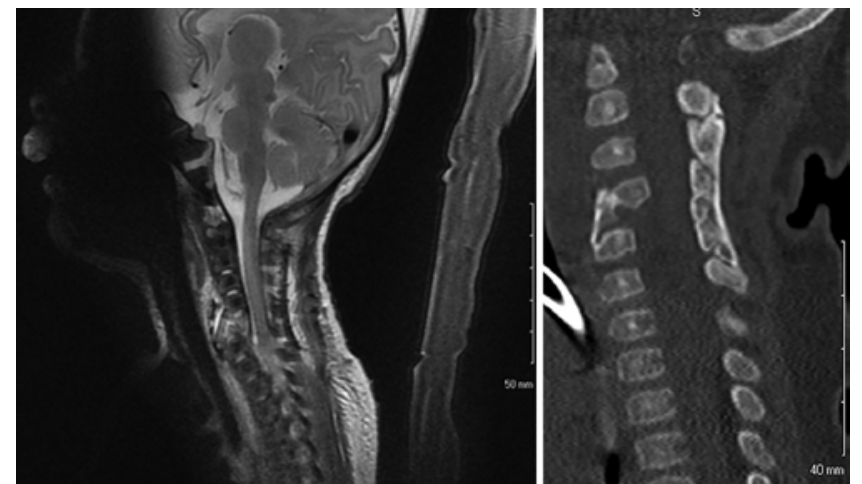

FIG. 3. Left: Postoperative sagittal T2-weighted cervical MR image demonstrating improved alignment of the spinal canal and decompression of the spinal cord. Right: Sagittal cervical CT demonstrating fusion 7 months after C-5 corpectomy and anterior C4-6 and posterior C2-7 arthrodesis with autologous rib graft.

dence of dissection. Follow-up CT imaging at 7 months demonstrated successful posterior bone fusion from C-4 to C-6 and bridging of bone anteriorly at these levels (Fig. 3 right).

Multiple attempts at extubation had failed, which were thought to be due to pulmonary hypertension due to the patient's abdominal ascites. After discussion with the pediatric critical care team, we elected to perform a tracheostomy for weaning the patient off the ventilator and conservative airway management, as there was concern about the difficulty with a potential need for reintubation given his Minerva brace and the limitations in neck motion allowed. The patient also required a Nissen fundoplication and gastrostomy placement for gastroesophageal reflux and continued poor oral nutritional intake, respectively.

The patient was discharged from the hospital with bilateral upper-extremity splints in addition to his custom Minerva brace, which was altered to allow for continued use of the gastrostomy and endotracheal tubes (Fig. 2). He underwent regular follow-up with physical and occupational therapy to improve function, especially in his hands. $\mathrm{He}$ also participated in speech therapy sessions frequently due to oral aversion, which led to delayed language development initially. The patient saw a physical medicine and rehabilitation physician every few months to coordinate care. He was weaned off the Minerva brace at 8 months, and his endotracheal tube was decannulated at 9 months. At 15 months, the patient began standing, and he was walking independently at 17 months. He was also using both hands at the time, with preferential use of his right hand due to remaining left-handed weakness in finger extension. At age 3.5 years, the patient was walking using orthotics, along with continued use of his gastrostomy tube for feeding due to ongoing oral weakness. It was also reported that the patient was speaking well at this age. He did begin to develop lumbar scoliosis due to his muscle weakness. At the latest follow-up by physical examination at the age of 4.5 years, the patient was found to be speaking well. His right wrist and fingers had a tendency to stay extended, while his left wrist and fingers remained flexed. He would use the tenodesis effect of wrist extension and flexion to aid in grasping and releasing, respectively. However, he was able to 
play with a smartphone by grasping it with the second and third fingers and using his thumb on the screen. His neck had a normal range of motion without pain, but the physical therapy team noted that the patient had impaired neck strength. He was able to mount, ride, and dismount a tricycle on his own and steer with his hands. The patient was using a thoracolumbosacral orthosis brace because of his scoliosis and orthotics, but he was walking independently over uneven surfaces.

\section{Discussion}

Although few cases of favorable outcomes of cervical dislocation after birth have been reported in the past,,$^{10,11,24}$ the incidence of such an injury is deemed to be higher than reported. ${ }^{1}$ In infants, the fulcrum of cervical spine movement is located at $\mathrm{C} 1-3$, leading to a majority of injuries in this region. Such injuries occur due to hyperflexion or hyperextension mechanisms. ${ }^{6,12}$ The nature of this patient's injury, being a bilateral C-5 pedicle fracture along with dislocation, indicates that the injury was likely a result of significant distractive force placed on the infant's head and neck during delivery. This is consistent with other studies and reports regarding cervical spine trauma in children. ${ }^{13,15,22}$ In the past, such maneuvers have been severe enough to result in decapitation, ${ }^{3,4}$ thus emphasizing the necessity of caution in cases of manual extraction when previously undiagnosed abdominal dystocia is encountered, including potentially switching to caesarean section for delivery. Furthermore, a high index of suspicion regarding possible trauma after a difficult delivery is important, and in this case might have led to this patient's cervical spine injury being identified earlier. ${ }^{2}$

Our patient currently exhibits improved functioning of all 4 limbs, in large part due to consistent, long-term, and multidisciplinary follow-up, including physical and occupational therapy. Good postoperative follow-up has been associated with favorable outcomes after cervical trauma repair. ${ }^{1}$ After successfully repairing the fracture and dislocation, our patient is walking and using his hands in part due to consistent physical and occupational therapy. He is speaking well and participating in school, despite his early oral aversion, due to regular speech therapy. Moreover, the lower location of his cervical injury may account for his successful response to surgery and improved neurological function, as higher cervical spine injuries have been associated with increased mortality. ${ }^{17}$ Furthermore, the lack of commercially available Minerva braces of such small size made working close with orthotics specialists essential. Ensuring an adequate design for stabilization and prevention of movement of the head and neck in any dimension was also critical for recovery. In addition, the brace required multiple custom openings for other complications the patient faced. Holes for gastrostomy and tracheostomy tubes were added, along with those for heat dissipation. Moreover, the patient's need for repeated imaging required that the brace be MRI compatible. Anticipating these possible needs in a medically complex patient facilitated his care and recovery by allowing uninterrupted stabilization of the cervical area by the Minerva brace.

We faced multiple surgical challenges given the patient's age and size. We had unsuccessfully attempted both a closed and open reduction of the dislocation prior to proceeding with the posterior fusion and $\mathrm{C}-5$ corpectomy. This was most likely due to the extent of injury to and displacement of components of the cervical spine. Instrumented fixation with standard titanium instrumentation was not possible because of the patient's young age, small size, and anticipated rapid growth, and therefore we had elected to use autologous structural rib graft sutured into position posteriorly with absorbable plate fixation used to augment the rib graft anteriorly. Rib grafts are associated with relatively low morbidity at the donor site and provide a good structural graft, which can match the natural curvature of the cervical spine. Moreover, a rib graft is easily harvested when performing the posterior portion of the procedure. The small size of the vertebrae also necessitated the use of smaller yet effective tools to perform the C-5 corpectomy. From previous experience in working with our otolaryngology colleagues, we had known smaller tools would be available in their surgical pans. We found an adequately small rongeur to best suit our need and were ultimately successful. Fortunately, the mini-absorbable plates available were of the appropriate size for the anterior fusion, although the structural stability provided by these absorbable plates is certainly much less than standard fixation and may not be significantly better than placement of the structural rib graft alone. Lastly, somatosensory evoked potential monitoring throughout the procedure was only achieved unilaterally due to the inconsistency in recordings from other nerves. While intraoperative neuromuscular monitoring is classically challenging in the pediatric population, especially in children younger than 3 years due to ongoing central and peripheral myelinization, it nonetheless may be useful in directing revisions during surgery. ${ }^{19,23}$ In our case, monitoring prompted us to perform a blood transfusion, which immediately improved the readings and may have prevented further harm in our patient.

Although cervical spine injuries are associated with high morbidity and mortality in both adult and pediatric populations,,$^{14,21}$ cases such as ours indicate the possibility of more favorable outcomes in children. ${ }^{8,10,11,18,24} \mathrm{We}$ illustrate that, with early identification, prompt action to surgically correct the injury, appropriate discharge conditions, and comprehensive follow-up, significant functional recovery after a spinal cord injury is possible in infants.

\section{References}

1. Babu RA, Arivazhagan A, Devi BI, Bhat DI, Sampath S, Chandramouli BA: Peculiarities and patterns of cervical spine injuries in children and adolescents: a retrospective series of 84 patients from a single institute. Pediatr Neurosurg 51:1-8, 2016

2. Caird MS, Reddy S, Ganley TJ, Drummond DS: Cervical spine fracture-dislocation birth injury: prevention, recognition, and implications for the orthopaedic surgeon. J Pediatr Orthop 25:484-486, 2005

3. Campbell JM: Traumatic decapitation and foetal ascites. Aust N Z J Obstet Gynaecol 8:22-25, 1968

4. Caulfield P: Missouri couple sues doctors for separating baby's head during grisly botched birth, and trying to cover it up: reports. New York Daily News. October 12, 2012. (http://www.nydailynews.com/life-style/health/couple-baby- 
decapitated-delivery-article-1.1181731) [Accessed September 13, 2017]

5. Cirak B, Ziegfeld S, Knight VM, Chang D, Avellino AM, Paidas CN: Spinal injuries in children. J Pediatr Surg 39:607-612, 2004

6. Easter JS, Barkin R, Rosen CL, Ban K: Cervical spine injuries in children, part II: management and special considerations. J Emerg Med 41:252-256, 2011

7. Eleraky MA, Theodore N, Adams M, Rekate HL, Sonntag VK: Pediatric cervical spine injuries: report of 102 cases and review of the literature. J Neurosurg 92 (1 Suppl):12-17, 2000

8. Farley FA, Hensinger RN, Herzenberg JE: Cervical spinal cord injury in children. J Spinal Disord 5:410-416, 1992

9. Ghatan S, Ellenbogen RG: Pediatric spine and spinal cord injury after inflicted trauma. Neurosurg Clin N Am 13:227-233, 2002

10. Gressot LV, Vadivelu S, Hwang SW, Fulkerson DH, Luerssen TG, Jea A: Spondylolysis of C-2 in children 3 years of age or younger: clinical presentation, radiographic findings, management, and outcomes with a minimum 12-month followup. J Neurosurg Pediatr 13:196-203, 2014

11. Ha SY, Lee SH, Kim ES, Shin HJ, Eoh W: Highly unstable cervical spine injury in an infant: a case report. Childs Nerv Syst 31:341-346, 2015

12. Hall DE, Boydston W: Pediatric neck injuries. Pediatr Rev 20:13-20, 1999

13. Holland CM, Kebriaei MA, Wrubel DM: Posterior cervical spinal fusion in a 3-week-old infant with a severe subaxial distraction injury. J Neurosurg Pediatr 17:353-356, 2016

14. Jain A, Brooks JT, Rao SS, Ain MC, Sponseller PD: Cervical fractures with associated spinal cord injury in children and adolescents: epidemiology, costs, and in-hospital mortality rates in 4418 patients. J Child Orthop 9:171-175, 2015

15. Klimo P Jr, Ware ML, Gupta N, Brockmeyer D: Cervical spine trauma in the pediatric patient. Neurosurg Clin N Am 18:599-620, 2007

16. Kokoska ER, Keller MS, Rallo MC, Weber TR: Characteristics of pediatric cervical spine injuries. J Pediatr Surg 36:100-105, 2001

17. Nitecki S, Moir CR: Predictive factors of the outcome of traumatic cervical spine fracture in children. J Pediatr Surg 29:1409-1411, 1994

18. Oluigbo CO, Gan YC, Sgouros S, Chapman S, Kay A, Solanki G, et al: Pattern, management and outcome of cervical spine injuries associated with head injuries in paediatric patients. Childs Nerv Syst 24:87-92, 2008
19. Pajewski TN, Arlet V, Phillips LH: Current approach on spinal cord monitoring: the point of view of the neurologist, the anesthesiologist and the spine surgeon. Eur Spine J 16 (Suppl 2):S115-S129, 2007

20. Platzer P, Jaindl M, Thalhammer G, Dittrich S, KutschaLissberg F, Vecsei V, et al: Cervical spine injuries in pediatric patients. J Trauma 62:389-396, 2007

21. Sribnick EA, Hoh DJ, Dhall SS: Traumatic high-grade cervical dislocation: treatment strategies and outcomes. World Neurosurg 82:1374-1379, 2014

22. Tekes A, Pinto PS, Huisman TA: Birth-related injury to the head and cervical spine in neonates. Magn Reson Imaging Clin N Am 19:777-790, viii, 2011

23. Thirumala PD, Bodily L, Tint D, Ward WT, Deeney VF, Crammond DJ, et al: Somatosensory-evoked potential monitoring during instrumented scoliosis corrective procedures: validity revisited. Spine J 14:1572-1580, 2014

24. Ul Haq I, Gururaj AK: Remarkable recovery in an infant presenting with extensive perinatal cervical cord injury. BMJ Case Rep 2012:2-4, 2012

\section{Disclosures}

The authors report no conflict of interest concerning the materials or methods used in this study or the findings specified in this paper.

\section{Author Contributions}

Conception and design: Swanson. Acquisition of data: Saleh, Swanson. Drafting the article: Saleh. Critically revising the article: all authors. Reviewed submitted version of manuscript: Swanson, Bragg. Study supervision: Bragg. Attending neurosurgeon: Bragg.

\section{Supplemental Information Current Affiliations}

Dr. Swanson: Aurora Neuroscience Innovation Institute, Milwaukee, WI.

\section{Correspondence}

Sara Saleh, Department of Neurological Surgery, University of Wisconsin School of Medicine and Public Health, 600 Highland Ave., Box 8660, Madison, WI 53792. email: ssaleh@wisc.edu. 